\title{
Sprachumstellung der Zeitschrift
}

\section{DOI 10.1007/s11576-014-0436-0}

\section{Der Autor}

Prof. Dr. Martin Bichler ( $₫)$

Decision Sciences \& Systems

Institut für Informatik

TU München

Boltzmannstraße 3

85748 München

Deutschland

bichler@in.tum.de

Online publiziert: 2014-10-17

This article is also available in English via http://www.springerlink.com and http://www.bise-journal.org: Bichler M (2014) Language Change. Bus Inf Syst Eng. doi: 10.1007/s12599-0140348-y.

(C) Springer Fachmedien Wiesbaden 2014
Wir möchten in diesem Rahmen noch einmal auf die Umstellung der Sprache der Zeitschrift im Jahr 2015 hinweisen. Die Umstellung wurde nach Diskussion und dem Beschluss in den Herausgebersitzungen im Jahr 2013 und 2014 über verschiedene Kanäle, in Editorials, E-Mails an die Abonnenten und auf der Homepage der Zeitschrift angekündigt.

Seit 2009 wird die Zeitschrift WIRTSCHAFTSINFORMATIK (56. Jahrgang) in die englische Sprache übersetzt und steht online als Business \& Information Systems Engineering (BISE) zur Verfügung. Dieser Schritt wurde im Rahmen der Triple-Strategie unternommen, um die Inhalte der Zeitschrift auch einer internationalen Leserschaft zugänglich zu machen. Neben diesen wissenschaftlich ausgerichteten Zeitschriften wurde 2009 die praxisorientierte Zeitschrift Wirtschaftsinformatik \& Management (WuM) ins Leben gerufen, um die unterschiedlichen Zielgruppen besser adressieren zu können.

Die englischsprachige BISE hat sich inzwischen international etabliert. Den erfolgreichen Weg der Internationalisierung haben wir im vergangenen Jahr mit einer neuen Struktur des Editorial Boards und internationaler Besetzung fortsetzen können. Als weiteren Schritt werden wir die Printversion der Zeitschrift ab dem Jahrgang 2015 auf Englisch publizieren. Auf die deutschsprachige Fassung wird insgesamt verzichtet - dies auch auf Wunsch vieler Autoren, für die der Aufwand der zweisprachigen Manuskripterstellung sehr hoch ist. Mit der WuM und der seit kurzem von Springer übernommenen Zeitschrift HMD - Praxis der Wirtschaftsinformatik stehen Ihnen nun ab 2015 gleich zwei alternative deutschsprachige Fachzeitschriften als Bezugsoption zur Verfügung. Sie können damit aus drei Zeitschriften, die sich inhaltlich ergänzen, auswählen. Wenn Sie derzeit die Zeitschrift WIRTSCHAFTSINFORMATIK beziehen, dann werden Sie ab 2015 die englischsprachige Business \& Information Systems Engineering erhalten. 